\title{
Pathogenicity Spectra and Screening for Resistance in Barley Against Tunisian Pyrenophora teres f. teres
}

Aida Bouajila and Néjia Zoghlami, Centre de Biotechnologie de Borj-Cédria, BP 901, Hammam-Lif 2050, Tunisia; Maha Al Ahmed and Michael Baum, International Center for Agricultural Research in the Dry Areas (ICARDA), Aleppo, Syria; Abdelwahed Ghorbel, Centre de Biotechnologie de Borj-Cédria; and Kumarse Nazari, ICARDA

\begin{abstract}
Bouajila, A., Zoghlami, N., Al Ahmed, M., Baum, M., Ghorbel, A., and Nazari, K. 2012. Pathogenicity spectra and screening for resistance in barley against Tunisian Pyrenophora teres f. teres. Plant Dis. 96:1569-1575.

This work aimed to determine patterns of pathogenicity in Pyrenophora teres f. teres and to identify potentially effective resistance sources that could be used as breeding material to control net blotch in Tunisia. Extensive pathogenic variability was detected in 85 isolates of $P$. teres causing net blotch of barley in Tunisia. Based on unweighted pair-group method with arithmetic averaging clustering and mean disease rating scores, three distinct virulence groups were identified. The isolates were classified into 23 pathotypes. Pathogenic variability

that can guide a rational choice of isolates for screening lines as part of a breeding program. Conversely, studying the relationship between geographic and pathotypic structure allowed us to detect a significant isolation by distance pattern, suggesting a regular and gradual dispersal of the pathogen over this spatial scale. Using specific resistance properties of individual barley genotypes as virulence markers, all the differential barley genotypes were shown to be distinct, and no single source of resistance was totally effective against all isolates.
\end{abstract} within the groups was higher than that between the groups, a finding
Pyrenophora teres Drechsler (anamorph: Drechslera teres (Sacc.) Shoemaker) is an ascomycete that causes net blotch in barley. This persistent disease is common in all barley-growing regions of the world and occurs typically in cool and humid areas. However, it is now a serious disease in the dry areas of North Africa, the Middle East, and Australia, and may cause up to $40 \%$ loss in grain yield $(18,28)$ in susceptible barley cultivars under epidemic conditions (30). Two forms of the disease, the net form ( $P$. teres $\mathrm{f}$. teres) and the spot form ( $P$. teres $\mathrm{f}$. maculata), were described based on the symptoms in barley $(8,41,50)$. The net form is more common in North Africa and the Middle East (26).

In Tunisia, net blotch is a serious constraint to barley production $(14,62)$. The disease has become increasingly severe because of increasingly intensive production, large-scale monoculture of only a few varieties, and an environment conducive for disease development (63). Rainfall can also enhance disease severity, net blotch being particularly severe in temperate regions of high rainfall and humidity (52).

Given the high level of variability in the P. teres f. teres-barley pathosystem, a better understanding of pathogenicity, epidemiology, and host-pathogen interactions is needed to breed more resistant cultivars (39). Both major genes and quantitative trait loci (QTL) for net blotch resistance have been identified in barley. Resistance to barley net blotch has been shown to be conferred (or conditioned) by one to three major genes, depending on the cultivars and pathotypes used $(5,28,31,54,59)$. Major gene resistance has been shown to be incompletely dominant (47), dominant $(33,35)$, recessive $(28)$, duplicated (CI9819 carries duplicate genes for resistance to $P$. teres [Rptl b and Rptl c]; 33,44), and complementary (31). Molecular mapping and QTL analyses have been used to identify chromosome locations and estimate the effects of

Corresponding author: N. Zoghlami, E-mail: zoghlami_n@yahoo.fr

Accepted for publication 6 May 2012.

http://dx.doi.org/10.1094/PDIS-01-11-0072-RE

This article is in the public domain and not copyrightable. It may be freely reprinted with customary crediting of the source. The American Phytopathological Society, 2012. barley net blotch resistance $(R)$ genes. Two genes (rpt.r and rpt.k) in the centromeric region of chromosome $6 \mathrm{H}$ were identified as conferring net-form net blotch resistance, with effects ranging from 10 to $83 \%(1,37,55)$.

Though durable resistance to $P$. teres f. teres may be difficult to achieve, Buchannon and McDonald (12) illustrated that Ethiopian barley cultivars, as well as others, are excellent sources of resistance to P. teres f. teres isolates from Canada, Mexico, and the United States. However, breeding programs following the gene-forgene model and other approaches are not documented.

Patterns of virulence (specific ability to overcome an $R$ gene; 57 ) have been elucidated in many $P$. teres f. teres populations worldwide $(4,12,24,25,27,49,54,56,61)$ possessing high levels of pathogenicity (ensemble of specific and nonspecific disease-causing abilities; 57). For example, Bjarko (6) separated the Middle Eastern $P$. teres $\mathrm{f}$. teres isolates into seven virulence types and Montana isolates into five virulence types. Harrabi (27) reported four groups among isolates from the Mediterranean region. However, pathogenicity characteristics of Tunisian net blotch isolates are not adequately documented.

The present study sought to (i) determine patterns of pathogenic variability in the Tunisian $P$. teres $\mathrm{f}$. teres population, (ii) gain knowledge of the population structure over our sampling spatial scale by assessing the correlation between geographic and pathotypic distances, and (iii) identify new sources of resistance useful in monitoring net blotch and strengthening future breeding projects for durable disease resistance in Tunisian barley cultivars.

\section{Materials and Methods}

Collection of $\boldsymbol{P}$. teres f. teres isolates. To derive pathogen isolates, barley leaves infected with $P$. teres f. teres were sampled from 19 locations throughout the major barley-growing areas in Tunisia during the 2009 season from plants of either 'Rihane' (43 isolates) or local barley landraces (42 isolates). All leaf samples were collected from naturally infected fields using the hierarchical sampling method described by McDonald et al. (40): one field population was sampled at each location, drawing a composite sample from eight circular sites of $1 \mathrm{~m}$ in diameter, each located along two parallel transects (with four sites per transect). The two transects and the collection sites along the transects were separated from the adjacent spot by $10 \mathrm{~m}$. This field design allowed a total 
sampling area of $408 \mathrm{~m}^{2}$ (12 by $34 \mathrm{~m}$ ). Ten infected leaves were sampled from different plants or tillers in a circular sweep of each sampling site. Leaf samples were placed in paper envelopes, air dried at room temperature for $48 \mathrm{~h}$, and stored at $6^{\circ} \mathrm{C}$ until required. Most collections were made from the northern subhumid to semiarid region (75 isolates), which is the largest barley-growing area in Tunisia, and the rest from the central region ( 3 isolates) and the southern regions ( 7 isolates) of the country. In all, 85 P. teres $\mathrm{f}$. teres isolates were sampled from 19 locations (Table 1).

Single-spore isolation and inoculum production. Leaves showing typical net blotch symptoms were cut into discs 5 to 10 $\mathrm{mm}$ in diameter, surface sterilized in $90 \%$ ethanol for $10 \mathrm{~s}$ and in $1 \% \mathrm{NaClO}$ for $60 \mathrm{~s}$, rinsed twice in sterile deionized water for 1 min each time, blotted, dried, and aseptically transferred to potato dextrose agar (PDA) plates. The plates were incubated at $20^{\circ} \mathrm{C}$ for several days under alternating cycles of $12 \mathrm{~h}$ of nearultraviolet light and $12 \mathrm{~h}$ of darkness. After 3 to 5 days, single conidia were transferred with a needle, while looking through a microscope, to fresh PDA plates and incubated for 2 weeks to induce sporulation.

Winter barley differential genotypes. To describe the spectrum of pathogenicity, three plants each of the following 14 differential lines, as described by Clifford and Jones (16), were inoculated with each isolate: 'CI05401', 'CI06311', 'CI09820', 'CI0739', 'CI01243', 'CI04795', 'CI04502', 'CI04979', 'Proctor', 'Code 65', 'CI09518', 'TENN-61-119', 'CI09214', and 'Arta'.

Inoculation and disease assessment. The differential cultivars were grown from seed sown at a rate of 8 to 10 seeds/pot in plastic pots $13 \mathrm{~cm}$ in diameter and filled with a soil-less potting mix. The pots were arranged in a complete randomized design with three replicates and kept in a greenhouse. The plants were grown under a regime of $14 \mathrm{~h}$ of light and $10 \mathrm{~h}$ of darkness and inoculated 14 to 16 days after sowing when at Zadoks growth stage 13 (63). The seedlings were sprayed with a spore suspension of $P$. teres $\mathrm{f}$. teres $\left(4 \times 10^{4}\right.$ spores $\left./ \mathrm{ml}\right)$ to which had been added $0.1 \mathrm{ml}$ of Tween 20 per $100 \mathrm{ml}$ of the suspension. The inoculated plants were kept for $48 \mathrm{~h}$ in darkness in a mist chamber at $12^{\circ} \mathrm{C}$ under high relative humidity, after which they were moved back to the greenhouse at $17^{\circ} \mathrm{C}$. The plants were watered two to three times daily, taking care to pour the water at the base to prevent cross-contamination.

At 9 days after inoculation, the differential cultivars (Table 1) were inoculated and scored for resistance as described by Harrabi and Kamel (27), Afanassenko et al. (2), and Khan (34), using the following scale: 0 , no visible symptoms; 1 , pinpoint lesions, no chlorosis; 2, slightly elongated lesions, slight chlorosis; 3 , elongated lesions crisscrossed with net-like venation, moderate chlorosis; and 4, well-developed lesions, extensive chlorosis and necrosis. Lesions rated 0,1 , or 2 were considered an indication of incompatibility (resistant reaction), and those rated 3 or 4 of compatibility (susceptible reaction). Six leaves from each pot were scored.

Data analysis. Vanderplank defined "virulence" as the ability of an isolate to overcome a specific $R$ gene, and considered "aggressiveness" to be the average nonspecific disease-causing ability of an isolate across a set of cultivars (57). He used the term "pathogenicity" (57) to refer to the ensemble of specific and nonspecific disease-causing abilities. In our study, $P$. teres f. teres virulence was measured in terms of specific reactions on several cultivars of the same host species $(11,27)$. By contrast, in our study, we used the term "virulence" to refer to the ensemble of specific diseasecausing abilities across a set of barley differential cultivars, as previously documented by Harrabi and Kamel (27). Per isolate, virulence was taken to be the mean disease rating (MDR) across all the differentials, a value of 3.0 or more indicating a highly virulent isolate (27) (Table 1).

A resistance/susceptibility (R/S) matrix was built, showing the reactions of the 14 differentials to each of the 85 isolates. Patterns of pathogenicity of Tunisian $P$. teres f. teres isolates were determined through cluster analysis of the pathotype matrix using the unweighted pair-group method with arithmetic averaging (UPGMA), using the Darwin software package (http://darwin. cirad.fr/darwin). The assignment of isolates to pathotypes was performed based on MDR scores and pairwise pathotypic similarities. Isolates having a similarity value $>90 \%$ were assumed to belong to the same pathotype. To determine virulence classes, MDR scores were separated using least significant differences (LSD; $P<$ 0.05 ) found with the analysis of variance (ANOVA) procedure of the XLSTAT software package (http://www.xlstat.com). The significance of variation among and within net blotch isolates was tested with ANOVA, using the PROC GLM procedure of SAS software (SAS Institute Inc.).

The relationship between geographic and pathotypic distances was assessed in order to determine whether there was evidence of isolation by distance (IBD). Pairwise pathotypic distances were computed using Darwin software for the 85 P. teres isolates. We chose the Manhattan distance, which is commonly used for continuous variables and is less sensitive to large differences than is the Euclidean distance. A matrix of pairwise geographic distances was built using CIRCE software (http://www.ign.fr). The correlation between both matrices was assessed with a Mantel test (45) using the XLSTAT program (http://www.xlstat.com) and assuming 10,000 permutations. The linear regression between pairwise geographic and pathotypic distances was plotted using Excel (Microsoft Office).

To identify new sources of resistance to net blotch in Tunisia, the 14 differential barley cultivars were compared for their response to the 85 P. teres $\mathrm{f}$. teres Tunisian isolates. R/S frequencies and mean isolate MDR score were calculated for each cultivar.

\section{Results}

Pathotypic variation. In total, 85 P. teres $\mathrm{f}$. teres isolates were tested for their pathogenicity against 14 differential barley cultivars (Table 1). The dendrogram constructed using UPGMA classified the 85 P. teres f. teres isolates into 23 pathotypes, among which 3 multi-strain pathotypes were the most prevalent: pathotypes 1 and 17 accounted for $16 \%$ of the isolates each, and pathotype 19 accounted for $19 \%$. The remaining pathotypes were represented by 1 to 10 isolates each.

In the low-, moderate-, and high-rainfall areas of Tunisia, 30, 40, and $22 \%$ of all isolates, respectively, belonged to unique pathotypes (i.e., only 1 isolate with that pathotype was identified in the total of 85 isolates).

Based on UPGMA clustering and MDR scores, three distinct virulence groups were identified. Indeed, Fisher's LSD analyses sorted isolates into three virulence groups (Table 1): group A, consisting of isolates with low MDR scores ( 0 to 1$)$; group $\mathrm{B}$, isolates with intermediate MDR scores (1 to 2.5); and group C, isolates with high MDR scores (2.5 to 3.8).

The clustering (Fig. 1) was independent of the eco-geographical origin of the isolates (host, extent of rainfall, and so on) but reflected the virulence spectra of the isolates. That is, all highly virulent pathotypes (Table 1) diverged significantly from the avirulent ones. Three major clusters (Fig. 1) were identified: cluster I contained the most virulent isolates (Table 1) and included nine pathotypes (P1 to P9); cluster II contained only isolate T9F1 (number 57 ), with intermediate virulence of 2.5 ; and cluster III contained most of the isolates with intermediate to low virulence. Pathotype 1 , which comprised 13 highly virulent isolates (with mean MDR of 3.5 ), was in the first cluster. Cluster III grouped together 13 pathotypes (P11 to P23). Cluster III included pathotype 19, which, with 16 isolates, was the largest (Fig. 1), and pathotype 18, which comprised the least virulent isolates, with a mean MDR score of 0.81 (Table 1). Isolates 56 (pathotype 10) and 57 (pathotype 11) were the closest isolates to $50 \% \mathrm{R} / \mathrm{S}$ (Fig. 1).

As indicated by ANOVA, the degree of pathogenic variability was significantly greater $(P<0.001)$ within the virulent group than between the groups (Table 2), a result that explains not only the high pathogenic divergence of isolates in their respective clusters but also the large number of subgroups in a cluster (Fig. 2). Therefore, all the isolates are equally important in screening for resistance to the pathogen. 
Table 1. Pathotypic variation in 85 Tunisian Pyrenophora teres isolates sampled in 2009 with their origin, climatic conditions, geographic position, and unweighted pair-group method with arithmetic averaging grouping ${ }^{\mathrm{a}}$

\begin{tabular}{|c|c|c|c|c|c|c|c|c|c|c|c|c|}
\hline Code & Isolate & $\begin{array}{l}\text { Host } \\
\text { cultivar }\end{array}$ & Origin/rain/eographic position ${ }^{c}$ & $\begin{array}{l}\text { CI05 } \\
401\end{array}$ & $\begin{array}{l}\text { CI06 } \\
311\end{array}$ & $\begin{array}{l}\text { CI09 } \\
820\end{array}$ & $\begin{array}{l}\text { CI00 } \\
739\end{array}$ & $\begin{array}{c}\text { CI01 } \\
243\end{array}$ & $\begin{array}{c}\text { CI04 } \\
795\end{array}$ & $\begin{array}{c}\text { CI04 } \\
502\end{array}$ & $\begin{array}{c}\text { CI49 } \\
79\end{array}$ & Proctor \\
\hline 1 & T1B1 & Rihane & Testour 1 (Béja), HR, NW & $\mathrm{R}$ & $\mathrm{R}$ & $\mathrm{R}$ & $\mathrm{R}$ & $\mathrm{R}$ & $\mathrm{R}$ & $\mathrm{R}$ & $\mathrm{R}$ & $\mathrm{R}$ \\
\hline 2 & $\mathrm{~T} 1 \mathrm{C} 2$ & Rihane & Testour 1 (Béja), HR, NW & $\mathrm{R}$ & $\mathrm{R}$ & $\mathrm{R}$ & $\mathrm{R}$ & $\mathrm{R}$ & $\mathrm{R}$ & $\mathrm{R}$ & $\mathrm{R}$ & $\mathrm{R}$ \\
\hline 3 & T1G1 & Rihane & Testour 1 (Béja), HR, NW & $\mathrm{R}$ & $\mathrm{R}$ & $\mathrm{R}$ & $\mathrm{R}$ & $\mathrm{R}$ & $\mathrm{R}$ & $\mathrm{R}$ & $\mathrm{R}$ & $\mathrm{R}$ \\
\hline 4 & $\mathrm{~T} 1 \mathrm{H} 1$ & Rihane & Testour 1 (Béja), HR, NW & $\mathrm{R}$ & $\mathrm{R}$ & $\mathrm{R}$ & $\mathrm{R}$ & $\mathrm{R}$ & $\mathrm{R}$ & $\mathrm{R}$ & $\mathrm{R}$ & $\mathrm{R}$ \\
\hline 5 & T16A1 & Rihane & Mosrata (Siliana), MR, NW & $\mathrm{R}$ & $\mathrm{R}$ & $\mathrm{R}$ & $\mathrm{R}$ & $\mathrm{R}$ & $\mathrm{R}$ & $\mathrm{R}$ & $\mathrm{R}$ & $\mathrm{R}$ \\
\hline 6 & T16H1 & Rihane & Mosrata (Siliana), MR, NW & $\mathrm{R}$ & $\mathrm{R}$ & $\mathrm{R}$ & $\mathrm{R}$ & $\mathrm{R}$ & $\mathrm{R}$ & $\mathrm{R}$ & $\mathrm{R}$ & $\mathrm{R}$ \\
\hline 7 & $\mathrm{~T} 17 \mathrm{C} 1$ & Rihane & Attayet (Siliana), MR, NW & $\mathrm{R}$ & $\mathrm{R}$ & $\mathrm{R}$ & $\mathrm{R}$ & $\mathrm{R}$ & $\mathrm{R}$ & $\mathrm{R}$ & $\mathrm{R}$ & $\mathrm{R}$ \\
\hline 8 & T17E1 & Rihane & Attayet (Siliana), MR, NW & $\mathrm{R}$ & $\mathrm{R}$ & $\mathrm{R}$ & $\mathrm{R}$ & $\mathrm{R}$ & $\mathrm{R}$ & $\mathrm{R}$ & $\mathrm{R}$ & $\mathrm{R}$ \\
\hline 9 & $\mathrm{~T} 17 \mathrm{~F} 3$ & Rihane & Attayet (Siliana), MR, NW & $\mathrm{R}$ & $\mathrm{R}$ & $\mathrm{R}$ & $\mathrm{R}$ & $\mathrm{R}$ & $\mathrm{R}$ & $\mathrm{R}$ & $\mathrm{R}$ & $\mathrm{R}$ \\
\hline 10 & T51E2 & Rihane & Bir Mcharga (Zaghouan), MR,NE & $\mathrm{R}$ & $\mathrm{R}$ & $\mathrm{R}$ & $\mathrm{R}$ & $\mathrm{R}$ & $\mathrm{R}$ & $\mathrm{R}$ & $\mathrm{R}$ & $\mathrm{R}$ \\
\hline 11 & $\mathrm{~T} 51 \mathrm{C} 2$ & Rihane & Bir Mcharga (Zaghouan), MR,NE & $\mathrm{R}$ & $\mathrm{R}$ & $\mathrm{R}$ & $\mathrm{R}$ & $\mathrm{R}$ & $\mathrm{R}$ & $\mathrm{R}$ & $\mathrm{R}$ & $\mathrm{R}$ \\
\hline 12 & $\mathrm{~T} 51 \mathrm{~F} 2$ & Rihane & Bir Mcharga (Zaghouan), MR,NE & $\mathrm{R}$ & $\mathrm{R}$ & $\mathrm{R}$ & $\mathrm{R}$ & $\mathrm{R}$ & $\mathrm{R}$ & $\mathrm{R}$ & $\mathrm{R}$ & $\mathrm{R}$ \\
\hline 13 & $\mathrm{~T} 52 \mathrm{~A} 1$ & Local & El fahs (Zagouhan), MR, NE & $\mathrm{R}$ & $\mathrm{R}$ & $\mathrm{R}$ & $\mathrm{R}$ & $\mathrm{R}$ & $\mathrm{R}$ & $\mathrm{R}$ & $\mathrm{R}$ & $\mathrm{R}$ \\
\hline 14 & T52B1 & Local & El fahs (Zagouhan), MR, NE & $\mathrm{R}$ & $\mathrm{R}$ & $\mathrm{R}$ & $\mathrm{R}$ & $\mathrm{R}$ & $\mathrm{R}$ & $\mathrm{R}$ & $\mathrm{R}$ & $\mathrm{R}$ \\
\hline 15 & $\mathrm{~T} 52 \mathrm{C} 1$ & Local & El fahs (Zagouhan) MR, NE & $\mathrm{R}$ & $\mathrm{R}$ & $\mathrm{R}$ & $\mathrm{R}$ & $\mathrm{R}$ & $\mathrm{R}$ & $\mathrm{R}$ & $\mathrm{R}$ & $\mathrm{R}$ \\
\hline 16 & T52D1 & Local & El fahs (Zagouhan), MR, NE & $\mathrm{R}$ & $\mathrm{R}$ & $\mathrm{R}$ & $\mathrm{R}$ & $\mathrm{R}$ & $\mathrm{R}$ & $\mathrm{R}$ & $\mathrm{R}$ & $\mathrm{R}$ \\
\hline 17 & T53D1 & Rihane & Saouaf (Zagouhan), MR, NE & $\mathrm{R}$ & $\mathrm{R}$ & $\mathrm{R}$ & $\mathrm{R}$ & $\mathrm{R}$ & $\mathrm{R}$ & $\mathrm{R}$ & $\mathrm{R}$ & $\mathrm{R}$ \\
\hline 18 & $\mathrm{~T} 53 \mathrm{~F} 2$ & Rihane & Saouaf (Zagouhan), MR, NE & $\mathrm{R}$ & $\mathrm{R}$ & $\mathrm{R}$ & $\mathrm{R}$ & $\mathrm{R}$ & $\mathrm{R}$ & $\mathrm{R}$ & $\mathrm{R}$ & $\mathrm{R}$ \\
\hline 19 & T55D1 & Rihane & Mograne (Zagouhan), MR, NE & $\mathrm{R}$ & $\mathrm{R}$ & $\mathrm{R}$ & $\mathrm{R}$ & $\mathrm{R}$ & $\mathrm{R}$ & $\mathrm{R}$ & $\mathrm{R}$ & $\mathrm{R}$ \\
\hline 20 & T57B1 & Rihane & Souidia (Bizerte), HR, NN & $\mathrm{R}$ & $\mathrm{R}$ & $\mathrm{R}$ & $\mathrm{R}$ & $\mathrm{R}$ & $\mathrm{R}$ & $\mathrm{R}$ & $\mathrm{R}$ & $\mathrm{R}$ \\
\hline 21 & $\mathrm{~T} 57 \mathrm{C} 1$ & Rihane & Souidia (Bizerte), HR, NN & $\mathrm{R}$ & $\mathrm{R}$ & $\mathrm{R}$ & $\mathrm{R}$ & $\mathrm{R}$ & $\mathrm{R}$ & $\mathrm{R}$ & $\mathrm{R}$ & $\mathrm{R}$ \\
\hline 22 & T57H1 & Rihane & Souidia (Bizerte), HR, NN & $\mathrm{R}$ & $\mathrm{R}$ & $\mathrm{R}$ & $\mathrm{R}$ & $\mathrm{R}$ & $\mathrm{R}$ & $\mathrm{R}$ & $\mathrm{R}$ & $\mathrm{R}$ \\
\hline 23 & T57A1 & Rihane & Souidia (Bizerte), HR, NN & $\mathrm{R}$ & $\mathrm{R}$ & $\mathrm{R}$ & $\mathrm{R}$ & $\mathrm{R}$ & $\mathrm{R}$ & $\mathrm{R}$ & $\mathrm{R}$ & $\mathrm{R}$ \\
\hline 24 & T59B1 & Rihane & Hamrounia (Bizerte), HR, NN & $\mathrm{R}$ & $\mathrm{R}$ & $\mathrm{R}$ & $\mathrm{R}$ & $\mathrm{R}$ & $\mathrm{R}$ & $\mathrm{R}$ & $\mathrm{R}$ & $\mathrm{R}$ \\
\hline 25 & T59G1 & Rihane & Hamrounia (Bizerte), HR, NN & $\mathrm{R}$ & $\mathrm{R}$ & $\mathrm{R}$ & $\mathrm{R}$ & $\mathrm{R}$ & $\mathrm{R}$ & $\mathrm{R}$ & $\mathrm{R}$ & $\mathrm{R}$ \\
\hline 26 & Т59H1 & Rihane & Hamrounia (Bizerte), HR, NN & $\mathrm{R}$ & $\mathrm{R}$ & $\mathrm{R}$ & $\mathrm{R}$ & $\mathrm{R}$ & $\mathrm{R}$ & $\mathrm{R}$ & $\mathrm{R}$ & $\mathrm{R}$ \\
\hline 27 & T1D1 & Rihane & Testour 1 (Béja), HR, NW & $\mathrm{R}$ & $\mathrm{R}$ & $\mathrm{R}$ & $\mathrm{R}$ & $\mathrm{R}$ & $\mathrm{R}$ & $\mathrm{s}$ & $\mathrm{R}$ & $\mathrm{R}$ \\
\hline 28 & $\mathrm{~T} 52 \mathrm{C} 2$ & Local & El fahs (Zagouhan), MR, NE & $\mathrm{R}$ & $\mathrm{R}$ & $\mathrm{s}$ & $\mathrm{R}$ & $\mathrm{R}$ & $\mathrm{R}$ & $\mathrm{R}$ & $\mathrm{R}$ & $\mathrm{R}$ \\
\hline 29 & $\mathrm{~T} 3 \mathrm{~F} 1$ & Rihane & Teboursek 1 (Béja), HR, NW & $\mathrm{R}$ & $\mathrm{R}$ & $\mathrm{R}$ & $\mathrm{R}$ & $\mathrm{R}$ & $\mathrm{R}$ & $\mathrm{R}$ & $\mathrm{R}$ & $\mathrm{R}$ \\
\hline 30 & T9G1 & Local & Barrage Maleg (Kef), MR, NW & $\mathrm{R}$ & $\mathrm{R}$ & $\mathrm{R}$ & $\mathrm{R}$ & $\mathrm{R}$ & $\mathrm{R}$ & $\mathrm{S}$ & $\mathrm{R}$ & $\mathrm{R}$ \\
\hline 31 & T9E1 & Local & Barrage Maleg (Kef), MR, NW & $\mathrm{R}$ & $\mathrm{R}$ & $\mathrm{R}$ & $\mathrm{R}$ & $\mathrm{R}$ & $\mathrm{R}$ & $\mathrm{s}$ & $\mathrm{R}$ & $\mathrm{R}$ \\
\hline 32 & $\mathrm{~T} 28 \mathrm{H} 1$ & Local & Hincha (Sfax), LR, S & $\mathrm{R}$ & $\mathrm{R}$ & $\mathrm{R}$ & $\mathrm{R}$ & $\mathrm{R}$ & $\mathrm{R}$ & $\mathrm{s}$ & $\mathrm{R}$ & $\mathrm{R}$ \\
\hline 33 & T51H1 & Rihane & Bir Mcharga (Zaghouan), MR,NE & $\mathrm{R}$ & $\mathrm{R}$ & $\mathrm{R}$ & $\mathrm{R}$ & $\mathrm{R}$ & $\mathrm{R}$ & $\mathrm{R}$ & $\mathrm{R}$ & $\mathrm{R}$ \\
\hline 34 & $\mathrm{~T} 51 \mathrm{H} 2$ & Rihane & Bir Mcharga (Zaghouan), MR,NE & $\mathrm{R}$ & $\mathrm{R}$ & $\mathrm{R}$ & $\mathrm{R}$ & $\mathrm{s}$ & $\mathrm{R}$ & $\mathrm{R}$ & $\mathrm{R}$ & $\mathrm{R}$ \\
\hline 35 & T51A2 & Rihane & Bir Mcharga (Zaghouan), MR,NE & $\mathrm{R}$ & $\mathrm{R}$ & $\mathrm{S}$ & $\mathrm{R}$ & $\mathrm{R}$ & $\mathrm{R}$ & $\mathrm{R}$ & $\mathrm{R}$ & $\mathrm{R}$ \\
\hline 36 & T53G3 & Rihane & Saouaf (Zagouhan), MR, NE & $\mathrm{R}$ & $\mathrm{R}$ & $\mathrm{s}$ & $\mathrm{R}$ & $\mathrm{R}$ & $\mathrm{R}$ & $\mathrm{R}$ & $\mathrm{R}$ & $\mathrm{R}$ \\
\hline 37 & $\mathrm{~T} 59 \mathrm{C} 1$ & Rihane & Hamrounia (Bizerte), HR, NN & $\mathrm{R}$ & $\mathrm{R}$ & $\mathrm{s}$ & $\mathrm{R}$ & $\mathrm{R}$ & $\mathrm{R}$ & $\mathrm{R}$ & $\mathrm{R}$ & $\mathrm{R}$ \\
\hline 38 & T16B1 & Rihane & Mosrata (Siliana), MR, NW & $\mathrm{R}$ & $\mathrm{R}$ & $\mathrm{s}$ & $\mathrm{R}$ & $\mathrm{S}$ & $\mathrm{R}$ & $\mathrm{R}$ & $\mathrm{R}$ & $\mathrm{R}$ \\
\hline 39 & $\mathrm{~T} 1 \mathrm{~F} 1$ & Rihane & Testour 1 (Béja), HR, NW & $\mathrm{R}$ & $\mathrm{R}$ & $\mathrm{R}$ & $\mathrm{R}$ & $\mathrm{R}$ & $\mathrm{R}$ & $\mathrm{R}$ & $\mathrm{R}$ & $\mathrm{R}$ \\
\hline 40 & T6E1 & Local & Bahra (Kef), MR, NW & $\mathrm{s}$ & $\mathrm{R}$ & $\mathrm{R}$ & $\mathrm{R}$ & $\mathrm{R}$ & $\mathrm{R}$ & $\mathrm{s}$ & $\mathrm{R}$ & $\mathrm{S}$ \\
\hline 41 & T6G1 & Local & Bahra (Kef), MR, NW & $\mathrm{R}$ & $\mathrm{s}$ & $\mathrm{R}$ & $\mathrm{R}$ & $\mathrm{s}$ & $\mathrm{R}$ & $\mathrm{s}$ & $\mathrm{R}$ & $\mathrm{R}$ \\
\hline 42 & $\mathrm{~T} 6 \mathrm{C} 1$ & Local & Bahra (Kef), MR, NW & $\mathrm{R}$ & $\mathrm{R}$ & $\mathrm{s}$ & $\mathrm{R}$ & $\mathrm{R}$ & $\mathrm{R}$ & $\mathrm{R}$ & $\mathrm{R}$ & $\mathrm{S}$ \\
\hline 43 & T52D2 & Local & El fahs (Zagouhan), MR, NE & $\mathrm{R}$ & $\mathrm{S}$ & $\mathrm{S}$ & $\mathrm{R}$ & $\mathrm{R}$ & $\mathrm{R}$ & $\mathrm{S}$ & $\mathrm{R}$ & $\mathrm{R}$ \\
\hline 44 & T15G1 & Local & Gboulat (Siliana), MR,NW & $\mathrm{R}$ & $\mathrm{R}$ & $\mathrm{R}$ & $\mathrm{S}$ & $\mathrm{R}$ & $\mathrm{R}$ & $\mathrm{R}$ & $\mathrm{s}$ & $\mathrm{R}$ \\
\hline 45 & T17D1 & Rihane & Attayet (Siliana), MR, NW & $\mathrm{R}$ & $\mathrm{R}$ & $\mathrm{s}$ & $\mathrm{R}$ & $\mathrm{R}$ & $\mathrm{R}$ & $\mathrm{R}$ & $\mathrm{s}$ & $\mathrm{R}$ \\
\hline 46 & T52E1 & Local & El fahs (Zagouhan), MR, NE & $\mathrm{R}$ & $\mathrm{R}$ & $\mathrm{S}$ & $\mathrm{R}$ & $\mathrm{R}$ & $\mathrm{S}$ & $\mathrm{R}$ & $\mathrm{R}$ & $\mathrm{R}$ \\
\hline 47 & T53B2 & Rihane & Saouaf (Zagouhan), MR, NE & $\mathrm{R}$ & $\mathrm{R}$ & $\mathrm{s}$ & $\mathrm{R}$ & $\mathrm{R}$ & $\mathrm{R}$ & $\mathrm{S}$ & $\mathrm{S}$ & $\mathrm{R}$ \\
\hline 48 & T3H1 & Rihane & Teboursek 1 (Béja), HR, NW & $\mathrm{s}$ & $\mathrm{R}$ & $\mathrm{s}$ & $\mathrm{R}$ & $\mathrm{R}$ & $\mathrm{R}$ & $\mathrm{R}$ & $\mathrm{R}$ & $\mathrm{R}$ \\
\hline 49 & T6D1 & Local & Bahra (Kef), MR, NW & $\mathrm{R}$ & $\mathrm{R}$ & $\mathrm{R}$ & $\mathrm{R}$ & $\mathrm{R}$ & $\mathrm{R}$ & $\mathrm{s}$ & $\mathrm{R}$ & $\mathrm{S}$ \\
\hline 50 & $\mathrm{~T} 28 \mathrm{G} 1$ & Local & Hincha (Sfax), LR, S & $\mathrm{R}$ & $\mathrm{s}$ & $\mathrm{R}$ & $\mathrm{R}$ & $\mathrm{S}$ & $\mathrm{R}$ & $\mathrm{s}$ & $\mathrm{R}$ & $\mathrm{R}$ \\
\hline 51 & T28A1 & Local & Hincha (Sfax), LR, S & $\mathrm{R}$ & $\mathrm{R}$ & $\mathrm{R}$ & $\mathrm{R}$ & $\mathrm{S}$ & $\mathrm{R}$ & $\mathrm{S}$ & $\mathrm{R}$ & $\mathrm{R}$ \\
\hline 52 & $\mathrm{~T} 51 \mathrm{C} 1$ & Rihane & Bir Mcharga (Zaghouan), MR,NE & $\mathrm{R}$ & $\mathrm{R}$ & $\mathrm{R}$ & $\mathrm{R}$ & $\mathrm{s}$ & $\mathrm{s}$ & $\mathrm{s}$ & $\mathrm{R}$ & $\mathrm{S}$ \\
\hline 53 & T62-A1 & Rihane & Utique (Bizerte), HR, NN & $\mathrm{s}$ & $\mathrm{S}$ & $\mathrm{s}$ & $\mathrm{R}$ & $\mathrm{R}$ & $\mathrm{s}$ & $\mathrm{R}$ & $\mathrm{R}$ & $\mathrm{R}$ \\
\hline 54 & T14B1 & Local & Touiref (Kef), MR, NW & $\mathrm{s}$ & $\mathrm{s}$ & $\mathrm{s}$ & $\mathrm{R}$ & $\mathrm{R}$ & $\mathrm{R}$ & $\mathrm{R}$ & $\mathrm{S}$ & $\mathrm{S}$ \\
\hline 55 & T14G1 & Local & Touiref (Kef), MR, NW & $\mathrm{s}$ & $\mathrm{s}$ & $\mathrm{R}$ & $\mathrm{R}$ & $\mathrm{s}$ & $\mathrm{R}$ & $\mathrm{s}$ & $\mathrm{R}$ & $\mathrm{R}$ \\
\hline 56 & $\mathrm{~T} 17 \mathrm{H} 1$ & Rihane & Attayet (Siliana), MR, NW & $\mathrm{s}$ & $\mathrm{s}$ & $\mathrm{R}$ & $\mathrm{S}$ & $\mathrm{s}$ & $\mathrm{s}$ & $\mathrm{s}$ & $\mathrm{R}$ & $\mathrm{R}$ \\
\hline 57 & $\mathrm{~T} 9 \mathrm{~F} 1$ & Local & Barrage Maleg (Kef), MR, NW & $\mathrm{R}$ & $\mathrm{S}$ & $\mathrm{s}$ & $\mathrm{R}$ & $\mathrm{S}$ & $\mathrm{R}$ & $\mathrm{S}$ & $\mathrm{S}$ & $\mathrm{S}$ \\
\hline 58 & T6A1 & Local & Bahra (Kef), MR, NW & $\mathrm{s}$ & $\mathrm{s}$ & $\mathrm{s}$ & $\mathrm{R}$ & $\mathrm{R}$ & $\mathrm{S}$ & $\mathrm{s}$ & $\mathrm{R}$ & $\mathrm{R}$ \\
\hline 59 & T16E1 & Rihane & Mosrata (Siliana), MR, NW & $\mathrm{S}$ & $\mathrm{R}$ & $\mathrm{R}$ & $\mathrm{S}$ & $\mathrm{S}$ & $\mathrm{S}$ & $\mathrm{S}$ & $\mathrm{R}$ & $\mathrm{R}$ \\
\hline 60 & T55G1 & Rihane & Mograne (Zagouhan), MR, NE & $\mathrm{s}$ & $\mathrm{R}$ & $\mathrm{s}$ & $\mathrm{S}$ & $\mathrm{s}$ & $\mathrm{s}$ & $\mathrm{S}$ & $\mathrm{S}$ & $\mathrm{S}$ \\
\hline 61 & T9H1 & Local & Barrage Maleg (Kef), MR, NW & $\mathrm{s}$ & $\mathrm{s}$ & $\mathrm{s}$ & $\mathrm{S}$ & $\mathrm{s}$ & $\mathrm{R}$ & $\mathrm{s}$ & $\mathrm{R}$ & $\mathrm{R}$ \\
\hline 62 & $\mathrm{~T} 25 \mathrm{~B} 2$ & Local & Sidi Mtir, Bouficha, LR, C & $\mathrm{s}$ & $\mathrm{S}$ & $\mathrm{s}$ & $\mathrm{S}$ & $\mathrm{S}$ & $\mathrm{s}$ & $\mathrm{s}$ & $\mathrm{R}$ & $\mathrm{R}$ \\
\hline 63 & $\mathrm{~T} 57 \mathrm{~F} 1$ & Rihane & Souidia (Bizerte), HR, N & $\mathrm{s}$ & $\mathrm{R}$ & $\mathrm{s}$ & $\mathrm{S}$ & $\mathrm{s}$ & $\mathrm{R}$ & $\mathrm{S}$ & $\mathrm{R}$ & $\mathrm{R}$ \\
\hline 64 & $\mathrm{~T} 53 \mathrm{H} 1$ & Rihane & Saouaf (Zagouhan), MR, NE & $\mathrm{R}$ & $\mathrm{S}$ & $\mathrm{S}$ & $\mathrm{S}$ & $\mathrm{S}$ & $\mathrm{R}$ & $\mathrm{S}$ & $\mathrm{S}$ & $\mathrm{R}$ \\
\hline 65 & T9B1 & Local & Barrage Maleg (Kef), MR, NW & $\mathrm{s}$ & $\mathrm{s}$ & $\mathrm{S}$ & $\mathrm{s}$ & $\mathrm{S}$ & $\mathrm{R}$ & $\mathrm{s}$ & $\mathrm{s}$ & $\mathrm{R}$ \\
\hline 66 & $\mathrm{~T} 55 \mathrm{C} 2$ & Rihane & Mograne (Zagouhan), MR, NE & $\mathrm{S}$ & $\mathrm{S}$ & $\mathrm{S}$ & $\mathrm{S}$ & $\mathrm{S}$ & $\mathrm{s}$ & $\mathrm{s}$ & $\mathrm{S}$ & $\mathrm{S}$ \\
\hline 67 & T19A1 & Local & Sers (Kef), MR, NW & $\mathrm{s}$ & $\mathrm{S}$ & $\mathrm{s}$ & $\mathrm{s}$ & $\mathrm{S}$ & $\mathrm{R}$ & $\mathrm{s}$ & $\mathrm{S}$ & $\mathrm{S}$ \\
\hline 68 & T19B1 & Local & Sers (Kef), MR, NW & $\mathrm{s}$ & $\mathrm{S}$ & $\mathrm{s}$ & $\mathrm{S}$ & $\mathrm{s}$ & $\mathrm{R}$ & $\mathrm{s}$ & $\mathrm{S}$ & $\mathrm{S}$ \\
\hline 69 & T19E1 & Local & Sers (Kef), MR, NW & $\mathrm{s}$ & $\mathrm{S}$ & $\mathrm{S}$ & $\mathrm{S}$ & $\mathrm{S}$ & $\mathrm{R}$ & $\mathrm{s}$ & $\mathrm{S}$ & $\mathrm{S}$ \\
\hline 70 & T6H1 & Local & Bahra (Kef), MR, NW & $\mathrm{S}$ & $\mathrm{s}$ & $\mathrm{s}$ & $\mathrm{S}$ & $\mathrm{s}$ & $\mathrm{S}$ & $\mathrm{s}$ & $\mathrm{S}$ & $\mathrm{R}$ \\
\hline 71 & T14B2 & Local & Touiref (Kef), MR, NW & $\mathrm{s}$ & $\mathrm{s}$ & $\mathrm{s}$ & $\mathrm{s}$ & $\mathrm{s}$ & $\mathrm{s}$ & $\mathrm{s}$ & $\mathrm{s}$ & $\mathrm{S}$ \\
\hline 72 & $\mathrm{~T} 25 \mathrm{~A} 1$ & Local & Sidi Mtir, Bouficha (Sousse), LR,C & $\mathrm{S}$ & $\mathrm{S}$ & $\mathrm{S}$ & $\mathrm{S}$ & $\mathrm{S}$ & $\mathrm{S}$ & $\mathrm{S}$ & $\mathrm{S}$ & $\mathrm{S}$ \\
\hline 73 & $\mathrm{~T} 15 \mathrm{~F} 1$ & Local & Gboulat (Siliana), MR, NW & $\mathrm{S}$ & $\mathrm{S}$ & $\mathrm{s}$ & $\mathrm{S}$ & $\mathrm{S}$ & $\mathrm{s}$ & $\mathrm{S}$ & $\mathrm{S}$ & $\mathrm{S}$ \\
\hline 74 & $\mathrm{~T} 15 \mathrm{D} 1$ & Local & Gboulat (Siliana), MR, NW & $\mathrm{s}$ & $\mathrm{s}$ & $\mathrm{s}$ & $\mathrm{S}$ & $\mathrm{s}$ & $\mathrm{s}$ & $\mathrm{s}$ & $\mathrm{s}$ & $\mathrm{S}$ \\
\hline 75 & $\mathrm{~T} 15 \mathrm{G} 2$ & Local & & $\mathrm{S}$ & $\mathrm{S}$ & $\mathrm{s}$ & $\mathrm{S}$ & $\mathrm{S}$ & $\mathrm{s}$ & $\mathrm{S}$ & $\mathrm{S}$ & $\mathrm{S}$ \\
\hline 76 & $\mathrm{~T} 28 \mathrm{~F} 1$ & Local & Hincha (Sfax), LR, S & $\mathrm{s}$ & $\mathrm{S}$ & $\mathrm{s}$ & $\mathrm{S}$ & $\mathrm{s}$ & $\mathrm{s}$ & $\mathrm{s}$ & $\mathrm{s}$ & $\mathrm{S}$ \\
\hline 77 & T9A1 & Local & Barrage Maleg (Kef), MR, NW & $\mathrm{S}$ & $\mathrm{S}$ & $\mathrm{S}$ & $\mathrm{S}$ & $\mathrm{S}$ & $\mathrm{s}$ & $\mathrm{S}$ & $\mathrm{S}$ & $\mathrm{S}$ \\
\hline 78 & $\mathrm{~T} 52 \mathrm{H} 2$ & Local & El fahs (Zagouhan), MR, NE & $\mathrm{s}$ & $\mathrm{s}$ & $\mathrm{s}$ & $\mathrm{S}$ & $\mathrm{s}$ & $\mathrm{s}$ & $\mathrm{s}$ & $\mathrm{S}$ & $\mathrm{S}$ \\
\hline 79 & T28D1 & Local & Hincha (Sfax), LR, S & S & S & S & S & $\mathrm{S}$ & S & S & S & $\mathrm{S}$ \\
\hline 80 & $\mathrm{~T} 28 \mathrm{D} 2$ & Local & Hincha (Sfax), LR, S & $\mathrm{S}$ & $\mathrm{S}$ & $\mathrm{s}$ & $\mathrm{S}$ & $\mathrm{S}$ & $\mathrm{S}$ & $\mathrm{S}$ & $\mathrm{S}$ & $\mathrm{S}$ \\
\hline 81 & $\mathrm{~T} 29 \mathrm{~A} 1$ & Local & Sfax peripherique 1 (Sfax), LR, S & $\mathrm{s}$ & $\mathrm{s}$ & $\mathrm{s}$ & $\mathrm{s}$ & $\mathrm{S}$ & $\mathrm{s}$ & $\mathrm{s}$ & $\mathrm{S}$ & $\mathrm{S}$ \\
\hline 82 & $\mathrm{~T} 25 \mathrm{C} 3$ & Local & Sidi Mtir, Bouficha (Sousse), LR, C & $\mathrm{S}$ & $\mathrm{S}$ & $\mathrm{S}$ & $\mathrm{S}$ & $\mathrm{S}$ & $\mathrm{s}$ & $\mathrm{S}$ & $\mathrm{S}$ & $\mathrm{S}$ \\
\hline 83 & T55A3 & Rihane & Mograne (Zagouhan), MR, NE & $\mathrm{S}$ & $\mathrm{S}$ & $\mathrm{s}$ & $\mathrm{S}$ & $\mathrm{S}$ & $\mathrm{S}$ & $\mathrm{S}$ & $\mathrm{S}$ & $\mathrm{S}$ \\
\hline 84 & $\mathrm{~T} 15 \mathrm{~A} 1$ & Local & Gboulat (Siliana), MR, NW & $\mathrm{s}$ & $\mathrm{s}$ & $\mathrm{s}$ & $\mathrm{S}$ & $\mathrm{s}$ & $\mathrm{s}$ & $\mathrm{s}$ & $\mathrm{s}$ & $\mathrm{s}$ \\
\hline 85 & $\mathrm{~T} 15 \mathrm{E} 1$ & Local & Gboulat (Siliana), MR,NW & $\mathrm{S}$ & $\mathrm{S}$ & $\mathrm{S}$ & $\mathrm{S}$ & $\mathrm{S}$ & $\mathrm{S}$ & $\mathrm{S}$ & $\mathrm{S}$ & $\mathrm{S}$ \\
\hline
\end{tabular}

${ }^{\mathrm{a}} \mathrm{R}=$ resistance and $\mathrm{S}=$ susceptibility

c Rain: LR, MR, and HR = low, moderate, and high rainfall, respectively. Geographic position: northwest (NW), northeast (NE), south (S), and central (C).

${ }^{\mathrm{d}}$ Mean disease rating. 
Evidence for IBD pattern. A significant positive correlation between pathotypic and geographic distances was revealed $(r=$ $0.216, P<0.0001)$. In addition, the linear regression between pairwise geographic and pathotypic distances was $y=0.001 x+0.542$ $\left(R^{2}=0.046 ; r=0.214\right)$. From the regression plot (Fig. 3), an IBD pattern was found among $P$. teres $\mathrm{f}$. teres isolates sampled across the country.

Resistance sources useful in monitoring net blotch. Most barley differential genotypes varied greatly in their susceptibility to different isolates of $P$. teres $\mathrm{f}$. teres based on the MDRs for genotypes of 3.28 to 1.23 (Fig. 2). Data on resistance and susceptibility indicated substantial differences among all the lines with respect to overall level of differential responses. Arta exhibited very little differential response, because it was susceptible to almost all the isolates. The lowest mean susceptibility frequency was observed in CI09214, TENN61-119, and CI04795.

None of the cultivars tested was highly resistant to all isolates. Except for CI04502, CI 09820, and Arta, which were the least effective, the other cultivars were moderately resistant. However, because CI09214 scored the lowest MDR of 1.32-a rating of 2 meant slightly elongated lesions - this cultivar possessed the most effective source of resistance (Fig. 2) among the 14 tested cultivars.

\section{Discussion}

Different isolates of the pathogen and differential barley genotypes were used to identify virulence profiles of Tunisian $P$. teres $\mathrm{f}$. teres, the causal agent of barley net blotch.

Variation in the pathogenicity of $P$. teres $f$. teres was first emphasized by Pon (43), and pathogenic spectra of its isolates from different geographical areas are well documented $(33,48)$. Nonetheless, this is the first report on the pathogenicity of Tunisian $P$. teres f. teres and the study has uncovered extensive pathogenic variability. All $85 P$. teres isolates varied greatly in pathogenicity.

All 85 P. teres isolates investigated were differentially pathogenic, and none of the barley cultivars examined was highly resistant to all isolates. High pathogenic variability in $P$. teres f. teres has also been reported from Australia, New Zealand, Russia, and
Canada-countries outside the possible centers of origin of barley diversity $(3,12,24,57)$. The extent of variation is not surprising, given the occurrence of the sexual state (41), which allows recombination between pathotypes.

The cluster analysis (Fig. 1) uses the 23 P. teres f. teres pathotypes to form three larger clusters. Pathotypes 1, 17, and 19 were made up of the widely prevalent isolates. In other studies, some authors identified more than three clusters. In fact, the pathogen is highly variable, with several virulence types (5).

The broad $P$. teres $\mathrm{f}$. teres pathogenicity spectrum detected in this study should be kept in mind when choosing isolates for use in a net-blotch screening program. It is clear that, although no single isolate will be adequate for such screening, pathotype 1 (comprising 13 isolates), which elicited susceptible reactions on all of the tested differential cultivars and, therefore, was the most virulent, would constitute a suitable sample for initial screening (Fig. 1).

Because all virulent pathotypes (cluster I) diverged significantly from those virulent on the fewest lines (cluster III) (Fig. 1) and because the degree of pathogenic variability within the identified groups was greater than that between the groups (Table 2), it is suggested that all the isolates are equally important and strongly recommended in a screening program that should include the 13 most highly virulent isolates found in subgroup P1 of cluster I (Fig. 1), the least virulent isolates (pathotype 18), and 20 other isolates that exhibited specific virulence to the differential CI09214, the most resistant cultivar in the current study (Fig. 2; Table 1). The resistance of this cultivar has also been confirmed in other studies $(3,12,17,24)$.

Table 2. Analysis of variance of the pathogenic response of 85 Tunisian Pyrenophora teres $\mathrm{f}$. teres isolates

\begin{tabular}{lrccc}
\hline $\begin{array}{l}\text { Source of } \\
\text { variation }\end{array}$ & df & $\begin{array}{c}\text { Sum of } \\
\text { squares }\end{array}$ & $\begin{array}{c}\text { Variance } \\
\text { components }\end{array}$ & $\begin{array}{c}\text { Pathotypic } \\
\text { variation }(\%)\end{array}$ \\
\hline Between groups & 1 & 0.5 & 0.0 & 0.00 \\
Within groups & 83 & 41.5 & 0.5 & 100.00 \\
Total & 84 & $\ldots$ & $\ldots$ & 42 \\
\hline
\end{tabular}

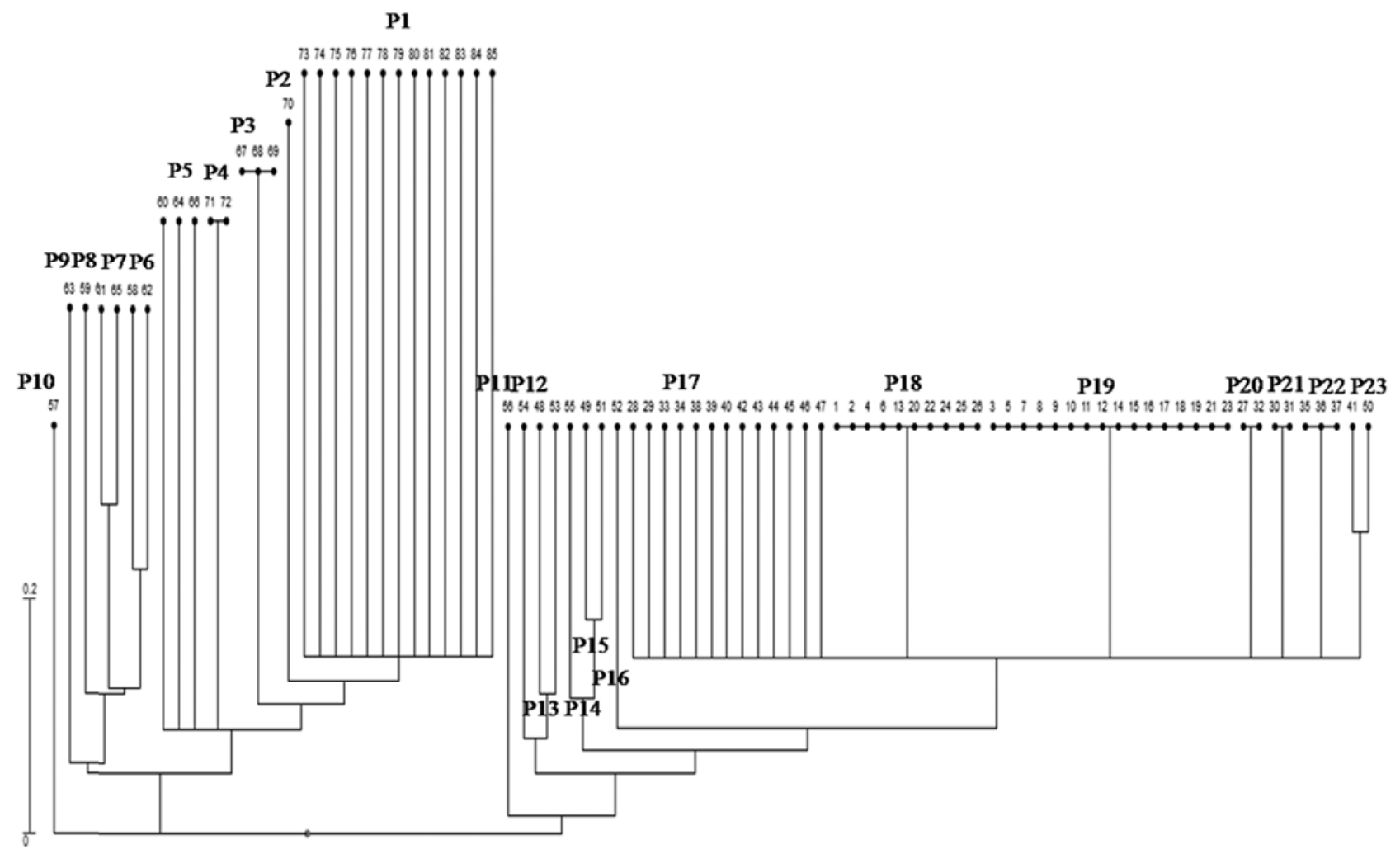

Fig. 1. Unweighted pair-group method with arithmetic averaging-derived dendrogram illustrating patterns of pathogenicity among 85 Tunisian Pyrenophora teres f. teres isolates based on their reaction spectra to 14 differential barley cultivars. P1 to P 23: pathotypes individualized. 
From the plot of the relationship between the pathotypic and geographic distances (Fig. 3), the main outcome of this study was the detection of an IBD pattern among $P$. teres $\mathrm{f}$. teres isolates. In addition, Mantel tests performed according to Rousset's method (45) were all significant. An IBD pattern may imply a regular and gradual dispersal of the pathogen over this spatial scale, rather than ubiquitous mixing, with gene flow decreasing over geographic distances.

The positive correlation between pathotypic and geographic distances might indicate that exchange of barley planting materials between different regions in Tunisia has contributed to a limited level of gene flow, as previously documented by Barrès et al. (6). Therefore, the population genetic structure of the pathogen would be conditioned by geographic distances rather than by the distribution of the host plant.

The detection of an IBD pattern greatly depends on the spatial scale studied $(13,45)$ but the appropriate sampling scale to reveal an IBD pattern for plant-pathogenic fungi is not obvious at first sight. This may account for the limited number of studies that detected IBD on plant pathogens $(10,19,23,40,64,65)$.

Using specific resistance properties of individual barley genotypes as virulence markers is common in examining the composition, diversity, and evolution of virulence in barley net blotch isolates and populations $(4,7,19,22,32,36,43,44,53)$. Knowledge of the reactions of putatively resistant cultivars and of the variability in the net blotch pathogen population is necessary for a successful resistance-breeding program. This study demonstrated that none of the tested genotypes was immune to infection. However, certain genotypes (CI09214, TENN61-119, and CI04795) showed a high resistance level compared with others (Fig. 2). Therefore, the resistance sources in the differential lines tested here will likely not confer durable resistance unless used in combination with other effective sources. Accordingly, it may be advantageous to incorporate suitable combinations of $R$ genes into barley adapted to Tunisia using recent discoveries regarding necrotrophic effectors $(38,42)$, inverse gene-for-gene interactions $(15,20)$, and transgressive segregation of $\mathrm{R} / \mathrm{S}$ analyses.

Recent work has shown that $P$. teres f. teres can produce host-selective toxins $(21,46,60)$. Incompatibility is due to the absence of either the toxin or the dominant allele of the host gene, and the result is resistance (if no other host-toxin interaction is present).
Therefore, this system (15) is essentially the inverse of the classic gene-for-gene system (20) at the host-toxin interface. However, the overall response of the host to a pathogen may not necessarily follow the inverse gene-for-gene model if multiple host-toxin interactions are involved because the interactions may show various effects such as additivity and epistasis, and other genes with minor effects may influence the overall response.

The transgressive segregation of $\mathrm{R} / \mathrm{S}$ can also reveal $R$ genes that are effective against $P$. teres $\mathrm{f}$. teres. For example, in the Arabidopsis-Leptosphaeria maculans pathosystem, transgressive segregation analyses revealed two Toll interleukin-1 receptor nucleotidebinding leucine-rich repeat $R$ genes against $L$. maculans fungus $(51,58)$. The segregation ratio close to $15: 1$ found in the $F_{2}$ population of a cross between two resistant lines, Col-0 and Ler-0, suggested that traits of two nonallelic $R$ genes, one from each parent, reside in each parent $(9,29)$.

Finally, the great variability detected in $P$. teres f. teres from Tunisia, the obvious relationship between pathogenicity and geographic origin, and the varieties that have been released as puta-

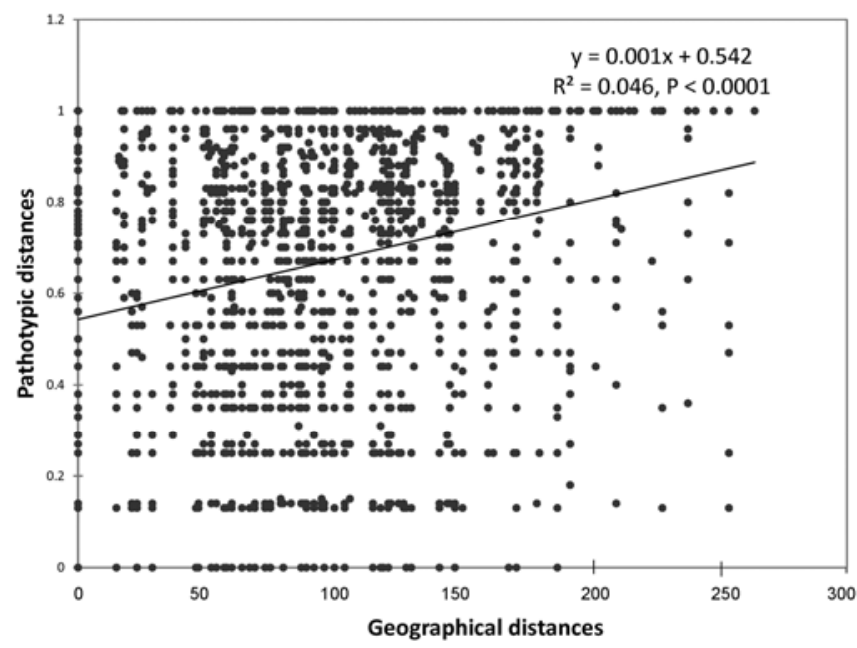

Fig. 3. Regression plot between pairwise pathotypic and geographic distances $P$ values were obtained by a Mantel test $(10,000$ permutations).

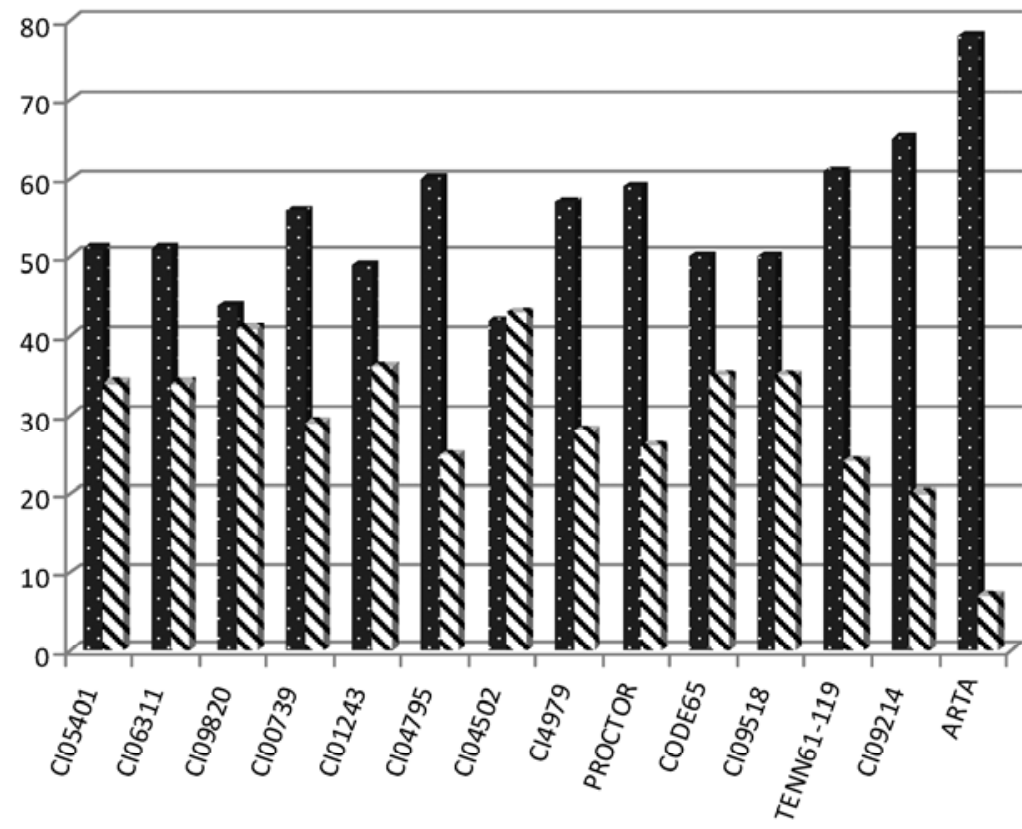

Fig. 2. Resistance/susceptibility frequencies among 14 tested barley differential cultivars challenged with 85 Tunisian Pyrenophora teres f. teres isolates, and mean disease rating values (numerals below cultivar names) based on a 0 -to- 4 scale: $0=$ no observable infection; $1=$ pinpoint lesions, no chlorosis; $2=$ slightly elongated lesions, slight chlorosis; 3 = elongated lesions, crisscrossed with netlike venation, moderate chlorosis; and 4 = well-developed lesions, extensive chlorosis and necrosis. 
tively resistant ought to be considered when determining strategies to breed resistant barley cultivars. For instance, effective sources of resistance to $P$. teres in CI09214, TENN61-119, and CI04795 was simply inherited, and incorporated into local barley cultivars that should be feasible. Selection of progeny based on resistance to current virulent $P$. teres f. teres pathotypes identified in this study may be useful. Conversely, marker-assisted selection consisting of molecular marker genotyping and DNA sequencing of interesting private fingerprints (bands) linked to resistance may also aid the identification of putative $R$ genes in CI09214. Genetic transformation procedures would subsequently facilitate their introgression in local barley cultivars.

\section{Acknowledgments}

This investigation was co-sponsored by ICARDA and the Ministry of Higher Education and Scientific Research in Tunisia. We thank the reviewers, especially C. Cowger, for doing hard work to provide very helpful reviews that greatly improved the manuscript.

\section{Literature Cited}

1. Abu Qamar, M., Liu, Z. H., Faris, J. D., Chao, S., Edwards, M. C., Lai, Z., Franckowiak, J. D., and Friesen, T. L. 2008. A region of barley chromosome $6 \mathrm{H}$ harbors multiple major genes associated with net type net blotch resistance. Theor. Appl. Genet. 117:1261-1270.

2. Afanasenko, O. S. 1977. The laboratory method of barley screening for resistance to net blotch. Agric. Biol. 12:297-299. (In Russian)

3. Afanasenko, O. S., Jalli, M., Pinnschmidt, H. O., Filatova, O., and Platz, G. J. 2009. Development of an international standard set of barley differential genotypes for Pyrenophora teres f. teres. Plant Pathol. 58:665-676.

4. Afanasenko, O. S., and Levitin, M. M. 1979. The population structure of the pathogen of net blotch of barley as regards its virulence. I. Identification of races. Mikol. Fitopatol. 13:230-234.

5. Arabi, M. I. E., Al-Safadi B., and Charbaji, T. 2003. Pathogenic variation among isolates of Pyrenophora teres, the causal agent of barley net blotch. J. Phytopathol. 151:376-382.

6. Barrès, B., Halkett, F., Dutech, C., Andrieux, A., Pinon, J., and Frey, P. 2008. Genetic structure of the poplar rust fungus Melampsora laricipopulina: evidence for isolation by distance in Europe and recent founder effects overseas. Infect. Genet. Evol. 8:577-587.

7. Bjarko, M. E. 1979. Sources and genetic action of resistance in barley to different virulent types of Pyrenophora teres, the causal organism of net blotch. Ph.D. thesis, Montana State University, Bozeman.

8. Bockelman, H. E., Sharp, E. L., and Bjarko, M. E. 1983. Isolates of Pyrenophora teres from Montana and the Mediterranean region that produce spot-type lesions on barley. Plant Dis. 67:696-700.

9. Bohman, S., Staal, J., Thomma, B. P. H. J., Wang, M., and Dixelius, C. 2004. Characterisation of an Arabidopsis Leptosphaeria maculans pathosystem: resistance partially requires camalexin biosynthesis and is independent of salicylic acid, ethylene and jasmonic acid signalling. Plant J. 37:9-20.

10. Brown, J. K. M., and Hovmøller, M. S. 2002. Aerial dispersal of pathogens on the global and continental scales and its impact on plant disease. Science 297:537-541.

11. Brown, J. S. 1990. Pathogenic variation among isolates of Rhynchosporium secalis from barley grass growing in southeastern Australia. Euphytica 50:81-89.

12. Buchannon, K. W., and McDonald W. C. 1965. Sources of resistance in barley to Pyrenophora teres. Can. J. Plant Sci. 45:189-193.

13. Castric, V., and Bernatchez, L. 2003. The rise and fall of isolation by distance in the anadromous brook charr (Salvelinus fontinalis Mitchill). Genetics 163:983-996.

14. Cherif, M., Harrabi, M., and Morjane, H. 1994. Distribution and importance of wheat and barley disease in Tunisia, 1989 to 1991. Rachis 13:25-34.

15. Chu, C. G., Faris, J. D., Xu, S. S., and Friesen, T. L. 2010. Genetic analysis of disease susceptibility contributed by the compatible Tsn1-SnToxA and Snn1-SnTox1 interactions in the wheat-Stagonospora nodorum pathosystem. Theor. Appl. Genet. 120:1451-1459.

16. Clifford, B. C., and Jones, D. 1981. Net blotch of barley. Pages 71-77 in: UK Cereal Pathogen Virulence 1980 Annual Report.

17. Cromey, M. G., and Parkes, R. A. 2003. Pathogenic variation in Drechslera teres in New Zealand. N. Z. Plant Prot. 56:251-256.

18. Daguenet, G. 1985. Essais fongicides sur orge dans le sud-ouest (19841985). Rapport I.T.C.F

19. Et-touil, K., Bernier, L., Beaulieu, J., Bérudé, J. A., Hopkin, A., and Hamelin, R. C. 1999. Genetic structure of Cronartium ribicola populations in eastern Canada. Phytopathology 89:915-919.

20. Flor, H. 1956. The complementary genetic systems in flax and flax rust. Adv. Genet. 8:29-54

21. Friesen, T. L., Faris, J. D., Solomon, P. S., and Oliver, R. P. 2008. Hostspecific toxins: effectors of necrotrophic pathogenicity. Cell. Microbiol. 10:1421-1428
22. Gacek, E. 1979. Studies on the resistance of barley to net blotch caused by Pyrenophora teres (Died.) Drechsl. Hodowla Ros. Aklim. Nasienn. 23:7383.

23. Gobbin, D., Rumbo, A., Linde, C. C., and Gessler, C. 2006. Population genetic structure of Plasmopara viticola after 125 years of colonization in European vineyards. Mol. Plant Pathol. 7:519-531.

24. Graner, A., Foroughi-Wehr, B., and Tekauz, A. 1996. RFLP mapping of a gene in barley conferring resistance to net blotch (Pyrenophora teres). Euphytica 91:229-234.

25. Grewal, T. S., Rossnagel, B. G., and Scoles, G. J. 2008. The utility of molecular markers for barley net blotch resistance across geographic regions. Crop Sci. 48:2321-2333.

26. Gupta, S., and Loughman, R. 2001. Current virulence of Pyrenophora teres on barley in Western Australia. Plant Dis. 85:960-966.

27. Harrabi, H. 1996. Breeding for resistance to the major fungal leaf pathogen of barley. Pages 265-275 in: Symposium Régional sur les Maladies des Céréales et des Légumineuses Alimentaires. B. Ezzahiri, A. Lyamani, A. Raih, and M. El Yamani, eds. Rabat, Maroc.

28. Harrabi, M., and Kamel, A. 1990. Virulence spectrum to barley in some isolates of Pyrenophora teres from the Mediterranean region. Plant Dis. 74:230-232.

29. Ho, K. M., Tekauz, A., Choo, T. M., and Martin, R. A. 1996. Genetic studies on net blotch resistance in a barley cross. Can. J. Plant Sci. 76:715-719.

30. Hovmoller, M. S., Caffier, V., Jalli, M., Andersen, O., Besenhofer, G., Czembor, J. H., Dreiseitl, A., Felsenstein, F., Fleck, A., Heinrics, F., Jonsson, R., Limpert, E., Mercer, P., Plesnik, S., Rashal, I., Skinnes, H., Slater, S., and Vronska, O. 2000. The European barley powdery mildew virulence survey and disease nursery 1993-1999. Agronomie 20:729-743.

31. Jayasena, K. W., van Burgel, A., Tanaka, K., Mejewski, J., and Loughman, R. 2007. Yield reduction in barley in relation to spot-type net blotch. Aust. Plant Pathol. 36:429-433.

32. Jonson, R., Sall, T., Kraft, T., Gustafsson, M. 1999. Inheritance of resistance to Pyrenophora teres f. sp. teres in spring barley. Plant Breed. 118:313-317.

33. Khan, T. N. 1971. Turkish barley varieties as a source of resistance to net blotch. Euphytica 20:292-298.

34. Khan, T. N. 1982. Changes in pathogenicity of Drechslera teres relating to changes in barley cultivars grown in Western Australia. Plant Dis. 66:655-656.

35. Khan, T. N., and Boyd, W. J. R. 1969. Physiologic specialization in Drechslera teres. Aust. J. Biol. Sci. 22:1229-1235.

36. Kolapudi, D. M. 1985. Epidemiological studies in relation to the breeding for resistance to net blotch (Drechslera teres (Sacc.) Shoem.) of barley (Hordeum vulgare L.). Ph.D. thesis, University of Western Australia, Nedlands, Australia.

37. Liu, Z., Justin, D., Faris, M., Edwards, C., and Friesen, T. L. 2010. Development of expressed sequence Tag (EST)-based markers for genomic analysis of a barley $6 \mathrm{H}$ region harboring multiple net form net blotch resistance genes. Plant Genome 3:41-52.

38. Liu, Z. H., Faris, J. D. Oliver, R. P., Tan, K. C., Solomon, P. S., McDonald, M. C., McDonald, B. A., Nunez, A., Lu, S., Rasmussen, J. B., and Friesen, T. L. 2009. SnTox3 acts in effector triggered susceptibility to induce disease on wheat carrying the Snn3 gene. PLoS Pathog. 5(9):E1000581. Online publication. doi:10.1371/journal.ppat.1000581

39. McDonald, B. A., and Linde, C. 2002. Pathogen population genetics, evolutionary potential and durable resistance. Annu. Rev. Phytopathol. 40:349 379.

40. McDonald, B. A., Zhan, J., and Burdon, J. J. 1999. Genetic structure of Rhynchosporium secalis in Australia. Phytopathology 89:639-645.

41. McLean, M. S., Barbara, J. H., and Hollaway, G. J. 2009. Epidemiology and control of spot form of net blotch (Pyrenophora teres $\mathrm{f}$. maculata) of barley: a review. Crop Pest Sci. 60:303-315.

42. Oliver, R. P., Rybak, K., Solomon, P. S., and Ferguson-Hunt, M. 2009. Prevalence of ToxA-sensitive alleles of the wheat gene Tsn1 in Australian and Chinese wheat cultivars. Crop Pest Sci. 60:348-352.

43. Pon, D. S. 1949. Physiologic specialization and variation in Helminthosporium teres.(Abstr.). Phytopathology 39:18.

44. Robinson, J., and Jalli, M. 1996. Diversity among Finnish net blotch isolates and resistance in barley. Euphytica 92:81-87.

45. Rousset, F. 1997. Genetic differentiation and estimation of gene flow from F statistics under isolation by distance. Genetics 145:1219-1228.

46. Sarpeleh, A., Wallwork, H., Tate, M. E., Catcheside, D. E. A., and Able, A. J. 2008. Initial characterization of phytotoxic proteins isolated from Pyrenophora teres. Phys. Mol. Plant Pathol. 72:73-79.

47. Schaller, C. W. 1955. Inheritance of resistance to net blotch in barley. Phytopathology 45:174-176.

48. Shipton, W. A., Khan, T. N., and Boyd, W. J. R. 1973. Net blotch of barley. Rev. Plant Pathol. 52:269-290.

49. Silvar, C., Casas, A. M., Kopahnke, D., Habekub, A., Schweizer, G., Gracia M. P., Lasa, J. M., Ciudad, F. J., Molina-Cano, J. L., Igartua, E., and Ordon, F. 2010. Screening the Spanish barley core collection for disease resistance. Plant Breed. 129:45-52.

50. Smedegaard-Petersen, V. 1971. Pyrenophora teres f. sp. maculata f. sp. nov. and Pyrenophora teres f. sp. teres on barley in Denmark. Pages 124-144 in: Yearbook of the Royal Veterinary and Agricultural University. Copenhagen.

51. Staal, J., Kaliff, M., Bohman, S., and Dixelius, C. 2006. Transgressive 
segregation reveals two Arabidopsis TIR-NB-LRR resistance genes effective against Leptosphaeria maculans, causal agent of blackleg disease. Plant J. 46:218-230.

52. Steffenson, B. J. 1997. Net blotch. Pages 28-31 in: Compendium of Barley Diseases, 2nd ed. D. E. Mather, ed. American Phytopathological Society, St. Paul, MN.

53. Steffenson, B. J. 1988. Investigations on Pyrenophora teres f. teres, the cause of net blotch of barley: pathotypes, host resistance, yield loss, and comparative epidemiology to Rhynchosporium secalis by time series analysis. Ph.D. thesis, University of California, Davis.

54. Steffenson, B. J., and Webster, R. K. 1992. Pathotype diversity of Pyrenophora teres $\mathrm{f}$. teres on barley. Phytopathology 82:170-177.

55. St. Pierre, S., Gustus, C., Steffenson, B., Dill-Macky, R., and Smith, K. P. 2010. Mapping net form net blotch and Septoria speckled leaf blotch resistance loci in barley. Phytopathology 100:80-84.

56. Tekauz, A. 1990. Characterization and distribution of pathogenic variation in Pyrenophora teres f. teres and P. teres f. maculate from western Canada. Can. J. Plant Pathol. 12:141-148.

57. VanderPlank J. E. 1968. Disease Resistance in Plants. Academic, New York (2nd ed., 1984, Academic, Orlando, FL).

58. West, J. S., Kharbanda, P. D., Barbetti, M. J., and Fitt, B. D. L. 2001. Epidemiology and management of Leptosphaeria maculans (Phoma stem canker) on oilseed rape in Australia, Canada and Europe. Plant Pathol. 50:10-27.

59. Williams, K. J., Lichon, A., Gianquitto, P., Kretschmer, J. M., Karakousis,
A., Manning, S., Langridge, P., and Wallwork, H. 1999. Identification and mapping of a gene conferring resistance to the spot form of net blotch (Pyrenophora teres f. sp. maculata) in barley. Theor. Appl. Genet. 99, 323327.

60. Wolpert, T. J., Dunkle, L. D., and Ciuffetti, L. M. 2002. Host-selective toxins and avirulence determinants: what's in a name? Annu. Rev. Phytopathol. 40:251-285.

61. Wu, H. L., Steffenson, B. J., Oleson, A. E., and Zhong, S. 2003. Genetic variation for virulence and RFLP markers in Pyrenophora teres. Can. J. Plant Pathol. 25:82-90.

62. Yahyaoui, A. H. 2004. Occurrence of barley leaf blights in Central Western Asia and North Africa. Pages 13-18 in: Meeting the Challenges of Barley Blights. Proc. 2nd Int. Workshop Barley Leaf Blights. A. H. Yahyaoui, L. Brader, A. Tekauz, H. Wallwork, and B. Steffenson, eds. ICARDA, Aleppo, Syria.

63. Zadoks, J. C., Chang, T. T., and Conzak, C. F. 1974. A decimal code for the growth stages of cereals. Weed Res. 14:415-421.

64. Zaffarano, P. L., McDonald, B. A., Zala, M., and Linde, C. C. 2006. Global hierarchical gene diversity analysis suggests the Fertile Crescent is not the center of origin of the barley scald pathogen Rhynchosporium secalis. Phytopathology 96:941-950.

65. Zeller, K. A., Bowden, R. L., and Leslie, J. F. 2004. Population differentiation and recombination in wheat scab populations of Gibberella zeae from the United States. Mol. Ecol. 13:563-571. 